\title{
Formações Discursivas na Comunicação Não Violenta
}

\author{
Formaciones discursivas en la comunicación no violenta
}

\section{Discursive Formations in Nonviolent Communication}

\author{
Sandra Barbosa Parzianello ${ }^{1}$
}

\begin{abstract}
Resumo
Propomos um debate à cerca das reflexões para se estabelecer práticas de Comunicação Não Violenta $(\mathrm{CNV})$ e Cultura de Paz, que dialoguem em torno dos saberes filosóficos e das Ciências Sociais. Partimos do pressuposto de que todo o ser humano social interage e interdepende do Outro, quando se dedica às soluções dialogadas e não impositivas. Nosso objetivo é valorizar e despertar sobre a importância do tema, bem como às novas dinâmicas das relações sociais do mundo contemporâneo em que os sujeitos venham a aprimorar as formas de relacionamento e convivência. Dada a ênfase da comunicação, como discurso, tomamos esta como constitutiva na promoção dos diálogos acerca das formas para a solução de conflitos, de modo a considerar no Outro, sua história, seus sonhos e seu olhar sobre o mundo, enfim, formações a partir das quais demonstram, como ferramenta metodológica a Comunicação Não Violenta (CNV), para a solução de litígios e manutenção da ética nos processos que norteiam essas novas ações comunicativas. Reiteramos com essa proposta, a importância sobre o fomento e produção do conhecimento que priorizam a comunicação interpessoal para a condução adequada dos conflitos. Portanto, nada mais relevante na contemporaneidade, do que a noção sobre a existência do Outro em nossas vidas e na formação de nossa identidade.
\end{abstract}

Palavras-chave: Comunicação; Linguagem; Formações Discursivas; Comunicação Não-Violenta; Cultura de Paz.

\section{Resumen}

Proponemos un debate a la cerca de las reflexiones para establecer prácticas de Comunicación No Violenta (CNV) y Cultura de Paz, que dialoguen en torno a los saberes filosóficos y de las Ciencias Sociales. Partimos del supuesto de que todo el ser humano social interactúa e interdepende del Otro, cuando se dedica a las soluciones dialogadas y no impositivas. Nuestro objetivo es valorar y despertar sobre la importancia del tema, así como a las nuevas dinámicas de las relaciones sociales del mundo contemporáneo en que los sujetos vengan a perfeccionar las formas de relación y convivencia. Dada el énfasis de la comunicación, como discurso, tomamos esta como constitutiva en la promoción de los diálogos acerca de las formas para la solución de conflictos, para considerar en el Otro, su historia, sus sueños y su mirada sobre el mundo, en fin, formaciones a partir de las cuales demuestran, como herramienta metodológica la Comunicación No Violenta (CNV), para la solución de litigios y mantenimiento de la ética en los procesos que orientan esas nuevas acciones comunicativas. Reiteramos con esa propuesta, la importancia sobre el fomento y producción del conocimiento que priorizan la comunicación interpersonal para la conducción adecuada de los conflictos. Por lo tanto, nada más relevante en la contemporaneidad, que la noción sobre la existencia del Otro en nuestras vidas y en la formación de nuestra identidad.

Palabras clave: Comunicación; idioma; Formaciones discursivas; Comunicación no violenta; Cultura de Paz.

\begin{abstract}
We propose a debate about the reflections to establish practices of Non-violent Communication (CNV) and Culture of Peace, which dialogue around the philosophical knowledge and Social Sciences. We start from the assumption that every social human being interacts and interdepends on the Other, when he engages in dialogue and non-taxation solutions. Our objective is to value and awaken the importance of the theme, as well as to the new dynamics of social relations of the contemporary world in which the subjects will improve the forms of relationship and coexistence. Given the emphasis of communication, as a discourse, we take this as a constitutive
\end{abstract}

\footnotetext{
${ }^{1}$ Doutoranda em Ciência Política (PPGCPol/UFPel); Pelotas; RS; Brasil; parzianellos@ yahoo.com.br
} 
one in promoting dialogues about ways to resolve conflicts, so as to consider in the "Other", its history, its dreams and its gaze on the world, from which they demonstrate, as a methodological tool the Nonviolent Communication (CNV), for the solution of litigation and ethical maintenance in the processes that guide these new communicative actions. We reiterate with this proposal, the importance on the promotion and production of knowledge that prioritize interpersonal communication for the proper conduct of conflicts. Therefore, nothing more relevant in the contemporary, than the notion about the existence of the Other in our lives and in the formation of our identity.

Keywords: Communication; Language; Discursive Formations; Non-Violent Communication; Culture of Peace.

\section{Introdução}

"O homem é um ser amarrado a teias e significados que ele mesmo teceu, sendo a cultura essas teias. Os indivíduos sentem, percebem, raciocinam, julgam e agem sob a direção destes símbolos. A experiência humana é assim significativa, interpretada e aprendida". (Glifford Gertz).

Comunicação Não Violenta (CNV) e Cultura de Paz são, hoje, dois temas sobre os quais se estabelece uma reflexão cada vez mais ampla nas Ciências Humanas. O debate na atualidade, reforça a importância, bem como as dimensões práticas, adaptada à realidade de formações discursivas que se desdobram a partir das relações humanas. Com base em estabelecer uma comunicação eficiente é que propomos esse trabalho, tendo como objetivo valorizar e despertar sobre a importância do tema, bem como novas dinâmicas das relações sociais no mundo contemporâneo em que os sujeitos possam aprimorar as formas de relacionamento e convivência.

Apontamos a linguagem, enquanto instrumento da comunicação que deve ser entendida aqui, em sua dimensão ontológica, portanto, constitutiva de uma essência e não simplesmente um instrumento de fala para o sujeito estabelecer contato com outrem. A partir do pensamento do filósofo francês, Michel Foucault (2014), a verdade é produzida pelo discurso, em que os elementos históricos são fundamentais para compreender a constituição da formação discursiva e dos discursos na sociedade contemporânea.

Tendo esse pensamento como estrutura fundamental tomamos a comunicação como instrumento da vida sistêmica, e tal como a linguagem, constitui o que somos a cada momento, em cada contingência, ainda que, expostos às precariedades que nos desestabilizam e que permitem novas possibilidade de significação. Em tempo, os sujeitos são o resultado das significações, que se revelam a partir das formações discursivas e das marcas que constituem nossas identidades.

Sendo assim, compreendemos ser oportuno pensar em valorizar e despertar sobre a importância do tema proposto, bem como às novas dinâmicas das relações sociais do mundo contemporâneo em que os sujeitos venham a aprimorar as formas de relacionamento e 
convivência, ao passo que a comunicação e a linguagem se apresentam como elementos determinantes para a construção da realidade em diferentes momentos e contingências.

É com base na comunicação que pretendemos refletir sobre o tema da $\mathrm{CNV}$ e da Cultura de Paz, tomando como princípio os fundamentos filosóficos e sob uma visão éticoprática. Atualmente, a CNV é uma ferramenta em potencial, para trabalhar os desafios da sociedade contemporânea que vem registrando cada vez mais conflitos de ordem negativa, nos mais diversos e diferentes ambientes como escolar, familiar e não obstante no âmbito judicial, pois, torna-se uma das Práticas Restaurativas.

Com efeito, a CNV torna-se um novo paradigma sob a perspectiva da cultura de paz, desde quando fundada pelo psicólogo, Marshall Rosenberg (2003)². A partir de então, a CNV insere-se em diferentes campos da sociedade e ao tornar sujeitos capacitados que almejam intervir e agir por meios práticos e eficientes, com o princípio de tomar o discurso a favor de diversos contextos em que a precariedade comunicativa persiste.

\section{Porque falar em formações discursivas?}

Ao tomarmos como base a comunicação, refletirmos em torno dos saberes filosóficos e das Ciências Humanas, para uma visão ético-prática, que desperte sobre a importância de determinados temas e, como estes tornam-se práticas revolucionárias nas relações sociais. A comunicação parte de determinado discurso ${ }^{3}$ que se constitui para a promoção de ações dialógicas acerca de formações discursivas cotidianas assim como para ações que possam despertar para a solução de conflitos, de modo a considerar no Outro, sua história, seus sonhos e seu olhar sobre o mundo. Em tempo, essas formações discursivas tornam-se ferramenta metodológica à Comunicação Não Violenta $(\mathrm{CNV})^{4}$ e por extensão a Cultura de Paz, para a solução de litígios e manutenção da ética nos processos que norteiam essas novas ações comunicativas.

Formações discursivas é uma das noções postas pelo filósofo Michel Foucault, ao refletir sobre os discursos, a partir de seu método arqueológico que, estrategicamente, questionou sobre como os discursos tornam-se verdadeiros. Ao mergulhar e perceber essas possibilidades de análises sobre as práticas discursivas construídas ao longo dos séculos

\footnotetext{
${ }^{2}$ ROSENBERG, Marshall. Nonviolent communication. A language of life. California: PuddleDancer Press, 2003. (traduzida no Brasil pela Editora Ágora, 2006).

3 Certamente os discursos são feitos de signos; mas o que fazem é mais que utilizar esses signos para designar coisas. É esse mais que os torna irredutíveis à língua e ao ato de fala. É esse "mais" que é preciso fazer aparecer e que é preciso descrever. (FOUCAULT, 2014, p. 60).

${ }^{4}$ A CNV se baseia em habilidades de linguagem e comunicação que fortalecem a capacidade de continuarmos humanos, mesmo em condições adversa. (ROSENBERG, 2006, p.21).
} 
XVIII e XIX pela teoria do conhecimento, reforçamos a importância e o papel que recai sobre o discurso que atravessa todos os elementos da experiência e está em todo conjunto de formas que comunicam um conteúdo.

Partimos dessa premissa para perceber como a comunicação está visivelmente entrelaçada a vida, aos enunciados e até mesmo ao ato e exercício do poder:

\begin{abstract}
Tal como nosso corpo é entendido como organismo, conjunto de membros, órgãos, tecidos, células, moléculas e energia, tal como construímos identidade apenas e desde sempre como família, parentalidade, sociabilidade e cultura, assim mostra-se o todo da comunicação em nossa vida. A comunicação, como linguagem, deve ser entendida primeiramente como dimensão ontológica (essencial, constitutiva), e não apenas instrumento para o ser humano entrar em contato com outrem por meio da fala. Comunicação é o fato de exercer a vida sistêmica, e tal como a linguagem, constitui o que somos a cada momento, a nossa história, as narrativas, memórias, feitos, sonhos, ideais, textos, discursos, enfim, signos. (PELIZZOLI, 2012, p. 1).
\end{abstract}

Com efeito, a teoria nos proporciona a percepção de que a linguagem não é simplesmente um meio pelo qual denominamos as coisas ou para comunicar, mas é também a forma de significação e construção da realidade. A realidade articulada, parte do inconsciente que se revela para seres que falam, seja pela forma de dizer e discursar ou até mesmo ao escrever. Portanto, a linguagem humana está enlaçada ao sujeito. Em tempo, é preciso enfatizar que o ponto de partida da linguística estruturalista está na ideia que a linguagem não pode ser limitada para as relações entre as palavras e as coisas. O estruturalismo, enquanto método de conhecimento e de caráter científico é limitado em suas aplicações e a determinado tratamento do homem em suas relações sociais.

Se tomarmos a concepção filosófica tradicional da linguagem, teremos uma visão de fato pobre e enganadora porque toma a linguagem como uma espécie de espelho das estruturas, da realidade ou da razão. Portanto, a diferença está em olhar o princípio do fundamento, sair do ente (fundamentado) ainda que de forma precária e refletir sobre o ser. O discurso filosófico ligado a uma noção ontológica pensa sempre o interno, pretende ser ele próprio um espelho, atitude essa que nega o devir. Porém, a partir dessa estrutura é que avançamos cientificamente, para propor possibilidades de articulação, que permitam pensar e explorar diferentes reflexões sobre a linguagem.

A partir dessa relação que se dá no campo da linguística, torna-se possível as possibilidades para o estudo das estruturas da linguagem como sistema dotado de princípios internos e de transformação das relações entre a linguagem e os outros sistemas de signos e símbolos ou outros sistemas de comunicação. Lembramos que a exclusão também é uma possibilidade do discurso, que está fora de uma enunciação discursiva, por aderir a outra forma e, ou, possibilidade. 
Para Foucault (2014) as formações discursivas compõem uma categoria fundamental para que possamos compreender o discurso em si que trata da construção linguística e gira em torno de um contexto social, o qual se pretende analisar, conforme vimos:

No caso em que se puder descrever, entre um certo número de enunciados, semelhante sistema de dispersão, e no caso em que entre os objetos, os tipos de enunciação, os conceitos, as escolhas temáticas, se puder definir uma regularidade (uma ordem, correlações, posições e funcionamentos, transformações), diremos, por convenção, que se trata de uma formação discursiva - evitando, assim, palavras demasiado carregadas de condições e consequências, inadequadas, aliás, para designar semelhante dispersão, tais como "ciência", ou "ideologia", ou "teoria", ou “domínio de objetividade”. (FOUCAULT, 2014, p. 47).

A formação discursiva dá-se em torno das condições em que se expressam os temas, os assuntos, suposições, enfim, de acordo com o discurso que, segundo Foucault (2013, p.10), “é, também, aquilo que é objeto do desejo; (...) aquilo porque, pelo que se luta, o poder do qual nos queremos apoderar." Foucault demonstra que, a partir das formações discursivas e dos enunciados é que ocorre a possibilidade de manter um discurso vivo, portanto, o discurso é uma articulação de enunciados, que exclui outros enunciados.

No sentido ontológico, enquanto um exercício filosófico, de crença que nos revela (onticamente) a presença da falta, conforme Oliver Marchart (2009). Notamos que, o que mais se invoca é um jogo infinito de diferenças vezes constituído por um imaginário, vezes constituído por um sujeito dividido e constituído pela falta. Sobre essa perspectiva o teórico argentino, pós-estruturalista, Ernesto Laclau, influenciado por Foucault, examina:

El gran avance llevado a cabo por el estructuralismo lue el reconocimiento del carácter relacional de toda identidad social; su límite lue la transfonn ación de estas relaciones en un sistema, en un objeto identilicable inteligible (es decir, en un esencia). Pero si mantenemos el carácter relacional de toda identidad y si, al mismo tiempo, renunciamos a Infijación de esas identidades en un sistema, en ese caso lo social debe ser identificado con el juego infinito de las diferencias, es decir, con lo que en el sentido más estricto del ténnino podemos llamar discurso — a condición, desde luego, de que liberemos al concepto de discurso de un significado que lo restringe al habla y a la escritura. (LACLAU, 2000, p. 104).

Teóricos pós-estruturalistas como Laclau, tomam o estruturalismo no sentido de pensar sobre a forma essencialista pensada naquele momento. O pós-estruturalismo é fundado por estruturalistas, portanto, o que se mantém forte e vivo é justamente a estrutura. Esse resguardo dá força a historicidade em uma temporalidade variável. Dessas experiências, que levaram a transição de um teórico estruturalista para pós-estruturalista, Laclau se diz otimista em relação aos movimentos sociais, o que leva a articular reflexos melhores ou piores, pois, dependerá dos sentidos articulados. 
Eni Puccinelli Orlandi ${ }^{5}$ (2001) também se apropria do pensamento foucaultiano quando afirma que com a linguística, a língua tem uma ordem nela mesma e que não se pode considerar que o sentido é um conteúdo depositado em algum lugar e que você vai procurar. Segundo Orlandi, o sentido está na materialidade discursiva, no fato de que a língua para significar tem que se inscrever na história. E reforça: “A formação discursiva se define como aquilo que numa formação ideológica dada - ou seja, a partir de uma posição dada em uma conjuntura sócio histórica dada - determina o que pode e deve ser dito”. (ORLANDI, 2001, p. 43).

Não se trata apenas de um acontecimento enquanto fato exterior ao discurso, mas parte constitutiva da discursividade, pois, segundo Orlandi, todo fato já é uma interpretação, sobretudo, o histórico que nos chega, via de regra, através da linguagem. Sua reflexão fica clara quando nos diz:

\begin{abstract}
Do mesmo modo podemos dizer que a evidência do sujeito, ou melhor, sua identidade (o fato de que "eu" sou "eu"), apaga o fato de que ela resulta de uma identificação: o sujeito se constitui por uma interpelação - que se dá ideologicamente pela sua inscrição em uma formação discursiva - que, em uma sociedade como a nossa produz sob a forma de sujeito de direito (jurídico). Esta forma de sujeito corresponde, historicamente, ao sujeito do capitalismo ao mesmo tempo determinado por condições externas e autônomo (responsável pelo que diz), um sujeito com seus direitos e deveres. (ORLANDI, 2001, p. 45).
\end{abstract}

Fundamentalmente, as relações sociais e humanas acabam sendo rígidas pela multiplicação dos discursos, por algo que chamamos de trocas, mas, também por dívidas e direitos, num verdadeiro jogo discursivo regido por algumas regras e pelas possibilidades em torno de demandas hegemonizadas.

Todo esse jogo de relações constitui um princípio de determinação que admite ou exclui, no interior de um dado discurso, um certo número de enunciados (...) uma formação discursiva não ocupa, assim todo o volume possível que lhe abrem por direito os sistemas de formação de seus objetos, de suas enunciações, de seus conceitos; ela é essencialmente lacunar, em virtude do sistema de formação de suas escolhas estratégicas. (...) uma dada formação discursiva pode fazer aparecerem possibilidades novas (...). (FOUCAULT, 2014, p. 79).

Percebemos então, à medida que o discurso se articula e provoca uma existência resultante de um processo, as formações discursivas podem se constituir, se manter ou mesmo serem esquecidas, em torno dos discursos contingentes. As condições específicas para uma dada identidade, podem pertencer há um momento e se estabelecer enquanto verdade, pois,

\footnotetext{
${ }^{5}$ Pesquisadora pioneira na esfera da análise do discurso (AD) de linha francesa no Brasil com suporte em Michel Pêcheux.
} 
RELACult - Revista Latino-Americana de Estudos em Cultura e Sociedade

Revista Latinoamericana de Estudios en Cultura y Sociedad | Latin American Journal of Studies in Culture and Society V. 05, ed. especial, abr., 2019, artigo n ${ }^{\circ} 1355$ | claec.org/relacult |e-ISSN: 2525-7870

muito se exige dos sujeitos enquanto responsabilidade, justiça social e verdades institucionalizadas, ainda que tenhamos verdades socialmente construídas.

\section{Em torno da Cultura, Comunicação e Cidadania}

Segundo o psicólogo Marshall Bertram Rosenberg (2006), a Comunicação NãoViolenta (CNV), apresenta-se como um método de resolução pacífica de conflitos, com base em técnicas comunicativas. Mas, o seu principal mérito consiste em ensinar e orientar a se colocar no lugar do outro através da empatia. Apropriando-se desse elemento, o psicólogo passou a enumerar diversos exemplos sociais da aplicação do seu método para a CNV, bem como a considerar valores comuns entre todos para que se estabeleça a comunicação, principalmente voltada a casos extremos de rupturas, desentendimentos e com aplicações na área educacional ao desenvolvimento de novos sistemas sociais, a fim de promover parcerias e o compartilhamento do poder.

No Brasil, esse método vem sendo aplicado há alguns anos, tornando-se um solo fértil na resolução de conflitos, assim como em mais de 65 países pelo mundo. Nosso país pertence a um grupo, ainda seleto e em desenvolvimento, onde também ocorre a aplicação do método em casos de Círculos Restaurativos e práticas de Justiça Restaurativa, a procura de sanar e promover a reconciliação e a solução pacífica das diferenças. Trata-se de um processo que facilita a comunicação interpessoal de modo mais intenso e cultiva o reconhecimento mútuo das necessidades afetivas profundas, ao levar compaixão e à solução pacífica de conflitos.

\footnotetext{
Mais do que qualquer outra coisa, a CNV é a tomada de consciência de nossas necessidades, nossa humanidade, nossa capacidade de conexão e nossa capacidade de comunicação, para além de qualquer linguagem rebuscada ou especulações gramaticais e lógicas. Igualmente, colhe sua força não de um constructo artificial utilitarista que vem sanar algo, mas sim das vontades prementes de entendimento, relação e superação de conflitos que habita em todo ser humano, salvo casos patológicos ou sociopatias empoderadas de modo incontornável. (PELIZZOLI, 2012, p. 2).
}

A CNV é um instrumento eficiente e oportuno para a capacitação de sujeitos sociais que, comprometidos com a o pensamento de uma Cultura de Paz, esperam (auto)educar em sociedade para estabelecer a confiança e o comprometimento de uma sociedade mais justa e comprometida entre sujeitos, instituições e as nações. Tomamos este método como uma via de (auto)reconhecimento.

Nessa linha de pensamento, compreendemos a importância de alguns eixos norteadores que corroboram na reflexão dessa proposta em sociedade, que gira em torno da cultura, da cidadania e da própria comunicação, que partem de um principal pressuposto: todo o ser humano social interage e interdepende do Outro. 
Julgamentos moralistas são péssimas expressões de necessidades, pois estes bloqueiam os indivíduos de ter consciência das necessidades suas que não estão sendo atendidas no relacionamento com o outro. Através desta cultura de julgamentos, os seres humanos são educados para pensar que necessitam de aprovação. Tanto julgamentos negativos quanto elogiosos levam a um distanciamento entre o que as pessoas são e desejam realmente. (MORAES, 2011).

Portanto, o que temos são formações discursivas que pertencem a cultura, porém, muitas vezes alguns profissionais do Direito são levados a atender demandas relacionadas a essas questões, o que eleva o número de processos sem resolução. Com o trabalho e técnica de conciliação, nos Círculos Restaurativos e nas práticas de Justiça Restaurativa, baseados no método da $\mathrm{CNV}$, é possível uma ação social eficiente (já comprovada em práticas por mediadores e conciliadores judiciais) tornando-se prática relevante que reduz, significativamente, o número de processos judiciais que tem se acumulado nos Fóruns de todo país.

Mas, valorizar e despertar sobre a importância dessa nova possibilidade de se estabelecer a comunicação é um trabalho que tem se revelado lento e desafiador, em especial entre profissionais que ainda desconhecem o tema, bem como sobre as novas dinâmicas para essas relações sociais que estão vingando no mundo contemporâneo, com o principal papel de aprimorar as relações entre os sujeitos e as formas de convivência.

Observar a sociedade a partir do viés cultural, enquanto um conjunto discursivo de todas as coisas as quais fazemos, ainda é um exercício a ser aprimorado. Compreendemos que no processo comunicacional, a teia cultural que é construída entre os homens em sociedade se estabelece por formações discursivas e pela forma como elaboramos. São essas formações discursivas que elaboram (pre)conceitos, equívocos sobre determinado assunto e revelam como, essencialmente, somos capazes ou, não, de compreender o outro, colocar-se no lugar outro.

A cidadania que buscamos pela $\mathrm{CNV}$ deixa de ser um mero conceito teórico, uma formação discursiva. A partir dessa compreensão, em busca de uma sociedade (plural) mais respeitosa e tolerante, pela empatia, devemos implicar no outro uma ação e valoração mútua. Desafiamos a compreender que a comunicação é a arte de interpretar o outro, de sermos sensíveis aos signos, a cultura e às formas de enunciar.

Façamos uso da linguagem, dos discursos para a construção de um mundo onde tudo que é tomado como fixo e limitado, tenha sua flexibilidade para o bem comum. Todos esses eixos norteadores devem colaborar enquanto estruturas que possam se articular e se entrelaçar, a fim de difundir um discurso consciente a favor da CNV e da Cultura de Paz, para que possamos pertencer a uma sociedade mais humana e solidária, mais justa e cidadã. 


\section{Considerações}

Apontamos para a pertinência desse estudo procurando também aprender a ser sujeitos comunicadores que, muitas vezes vivem em "mundos" paralelos ao sistema e às instituições, mas, que tem muito a contribuir para o desenvolvimento de uma Cultura de Paz, a partir de nosso próprio momento. Nota-se a ampliação e a articulação dos movimentos sociais e políticos (como nas campanhas eleitorais) dando relevância a essas práticas e métodos.

A CNV e a Cultura de Paz, nada mais são do que estratégias democráticas para a construção de algo que já é legítimo e que em nossa contemporaneidade se torna visível, notável graças a representatividade por grupos, a difusão pela evolução de paradigmas e a promoção de novos sentidos para a sociedade, num processo que arrisco chamar de (re)politização. Por isso, nos lançamos ao debate em torno do tema, sem deixar de considerar a contingência, o momento a precariedade da sociedade contemporânea.

Efetivamente, tratamos de um tema difícil, pois, exige conhecimento e esforço para a mudança, como um exercício para reaprender a comunicar-se. Todo processo de mudança é tenso e até agressivo em relação ao que estamos condicionados a aceitar. Porém, a possibilidade existe basta querer, compreender que antagonismos fazem parte de nosso mundo, que conseguir entender o que o outro quer, exige um esforço maior. Tomara que cada vez mais tenhamos agentes para uma CNV e para a Cultura de Paz, para perceber o outro, as possibilidades para as relações sociais, comunicacionais e notar que o mundo é vasto e próspero quando estamos prontos para articulá-lo junto com os outros.

\section{Referências}

FOUCAULT, Michel. A ordem do discurso: Aula inaugural no Collège de France. 23.ed. São Paulo: Edições Loyola, 2013.

A Arqueologia do Saber. 5.ed. Rio de Janeiro: Forense Universitária,

2014

LACLAU, Ernesto. Nuevas reflexiones sobre la revolución de nuestro tiempo. In: . Nuevas reflexiones sobre la revolución de nuestro tiempo. Buenos Aires:

Nueva Visión, 2000.

MARCHART, Oliver. El pensamiento político posfundacional: la diferencia política em Nancy, Lefort, Badiou y Laclau. Buenos Aires, Fondo de Cultura Económica, 2009. 
MORAES, Maria Carolina Fernandes; PELIZZOLI, Marcelo. Comunicação não-violenta (CNV): uma ética prática pela resolução de conflitos e empatia. Disponível em: http://pt.scribd.com/doc/22270801/CNV-Carol-e-Marcelo. Acesso em: 23 Dez. 2018.

ORLANDI, Eni Puccinelli. Análise do Discurso: princípios e procedimentos. 3.ed. Campinas, SP: Pontes, 2001.

PELIZZOLI, Marcelo L. Introdução à Comunicação Não Violenta (CNV) - reflexões sobre fundamentos e método. In: (Org.) Diálogo, mediação e cultura de paz. Recife: Ed. da UFPE, 2012.

ROSENBERG, Marshall B. Comunicação não-violenta: técnicas para aprimorar relacionamentos pessoais e profissionais. 2.ed. São Paulo: Ágora, 2006. 